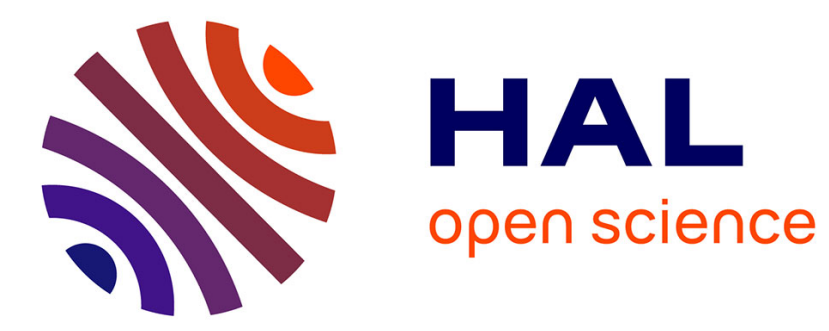

\title{
Undulators and wigglers for the new generation of synchrotron sources
}

\author{
P. Elleaume
}

\section{To cite this version:}

P. Elleaume. Undulators and wigglers for the new generation of synchrotron sources. Journal de Physique IV Proceedings, 1994, 04 (C9), pp.C9-3-C9-10. 10.1051/jp4:1994901 • jpa-00253461

\section{HAL Id: jpa-00253461 https://hal.science/jpa-00253461}

Submitted on 1 Jan 1994

HAL is a multi-disciplinary open access archive for the deposit and dissemination of scientific research documents, whether they are published or not. The documents may come from teaching and research institutions in France or abroad, or from public or private research centers.
L'archive ouverte pluridisciplinaire HAL, est destinée au dépôt et à la diffusion de documents scientifiques de niveau recherche, publiés ou non, émanant des établissements d'enseignement et de recherche français ou étrangers, des laboratoires publics ou privés. 


\section{Undulators and wigglers for the new generation of synchrotron sources}

P. Elleaume

ESRF, BP. 220, 38043 Grenoble cedex, France

\section{Abstract :}

The technological issues involved in the manufacturing of undulators and wigglers for the new generation of synchrotron radiation sources are discussed, with a particular emphasis on the solutions adopted at ESRF such as segmentation and shimming. The main characteristics of the radiation from the undulators and wigglers are discussed in term of total power, angular power, spectral brilliance and spectral flux. Illustration is again taken from ESRF. A brief description is given of the new devices that have been or can be, built to produce circularly polarized radiation.

\section{INTRODUCTION}

A large number of new synchrotron sources are being built all over the world. They include the ESRF (Grenoble France), APS (Argonne, USA) and Spring-8 (Japan) for the hard x-ray range of the spectrum , ALS (Berkeley, USA), Elettra (Trieste, Italy), SRRC (Taiwan) BESSY-II (Berlin, Germany) MAX-II (Sweden) for the soft $\mathrm{x}$-ray range of the spectrum.

These sources are optimized to gain full benefit from the insertion devices (ID) also called undulators and wigglers. They provide a small horizontal and vertical emittance and a large number of long straight sections with typical length of 3 to $6 \mathrm{~m}$. In the following I shall give a brief description of the technology of the IDs followed by the presentation of the characteristics of the radiation and give a few words on the generation of circularly polarized radiation. Illustration is mostly taken from ESRF. Today, the measured emittances are $8 \mathrm{~nm}$ horizontally and $0.2 \mathrm{~nm}$ vertically with a stored current of $100 \mathrm{~mA}$. The power, flux and brilliance given below assume the $200 \mathrm{~mA}$ current available in the short future.

\section{TECHNOLOGY OF INSERTION DEVICES}

The technologies available to build insertion devices include essentially permanent magnets, room temperature and super conducting electromagnets. The most economical choice is the room temperature electromagnet. However, room temperature electromagnets produce a significantly smaller magnetic field than permanent magnets at small periods over magnetic gap ratio. Since most undulators aim at the highest possible energy of the fundamental, the highest magnetic field at the shortest period possible is an absolute necessity. Consequently most designers prefer the use of permanent magnets. Today more than $90 \%$ of the undulators and wigglers built are made of permanent magnets. The permanent magnets used 
in the early times were made of Samarium and Cobalt alloys. Since then Neodymium Iron Boron $(\mathrm{NdFeB})$ alloys have replaced them in the majority of the systems. NdFeB magnets present a $10 \%$ to $20 \%$ higher magnetization, they are less brittle and cheaper to produce, however they are more sensitive to temperature, moisture and radiation damage. One typically distinguishes two classes of permanent magnet IDs, the so-called pure permanent magnet arrays and the hybrid arrays in which iron poles are inserted between the permanent magnet blocks. The hybrid technology gives a slightly higher magnetic field. In addition the magnetic field imperfections seen by the electrons are less sensitive to the inhomogeneities of the magnetization of the blocks. However pure permanent magnet technology is less expensive and 3D field simulations are trivial allowing a higher predictability of the magnetic field. At ESRF both technologies are used. The majority of the undulators use the pure permanent magnet technology while the wigglers use the hybrid technology.

The main difficulty in the manufacture of Insertion Devices is the tight specifications for the magnetic field. In order to minimize the perturbation to the closed orbit of the electrons in the storage ring, both the horizontal and vertical field integrated along the electron beam path must be as small as possible. Typical values as small as $10^{-4}$ to $10^{-5}$ Tesla $\mathrm{x}$ meter are required. The Brilliance of undulator is quite sensitive to the field periodicity. Peak to peak field fluctuations smaller than $0.5 \%$ are desirable. These field tolerances are difficult to meet with permanent magnets. The systematic removal of the field imperfections results in a typical high cost of the IDs when compare to the cost of dipole and quadrupole magnets constituting the storage ring lattice. In order to achieve these narrow tolerances, one must use magnet blocks as uniform as possible in term of orientation, absolute value and homogeneity of the magnetization. The residual differences from block to block must be compensated by a careful measurement and pairing in order to compensate most of the field errors over one or a few period of the magnetic field. To further improve the field quality a shimming[1] can be applied. The shimming consists in placing thin $(0.1$ to $0.2 \mathrm{~mm})$ iron plates on the surface of the magnetic array at specific places. These plates are magnetized by the surrounding magnetic blocks and modify the magnetic field in the air in such a way that they compensate the field errors induced by the imperfect blocks. This shimming technique has been intensively developed at ESRF and routinely applied to more than 17 Undulator and wiggler segments. It has resulted in a dramatic reduction of the production cost.

The magnetic field seen by the electrons needs to be changed according to the desired photon energy. This is accomplished by varying the magnetic gap between the upper and lower magnet arrays in between which the electron beam is propagated. Since magnetic forces as high as 10 to 20 tons can occur between these arrays, a rigid high precision mobile carriage is required. Most straight section of the storage rings of the new generation are 5 meters long. To limit the cost and difficulty in handling and measuring long single piece insertion devices, a segmented approach has been initiated at ESRF and followed by several other laboratories (TRIESTE, SPRING-8). Figure 1 presents a view of a 5 meters long ESRF straight section equipped with three segments. 


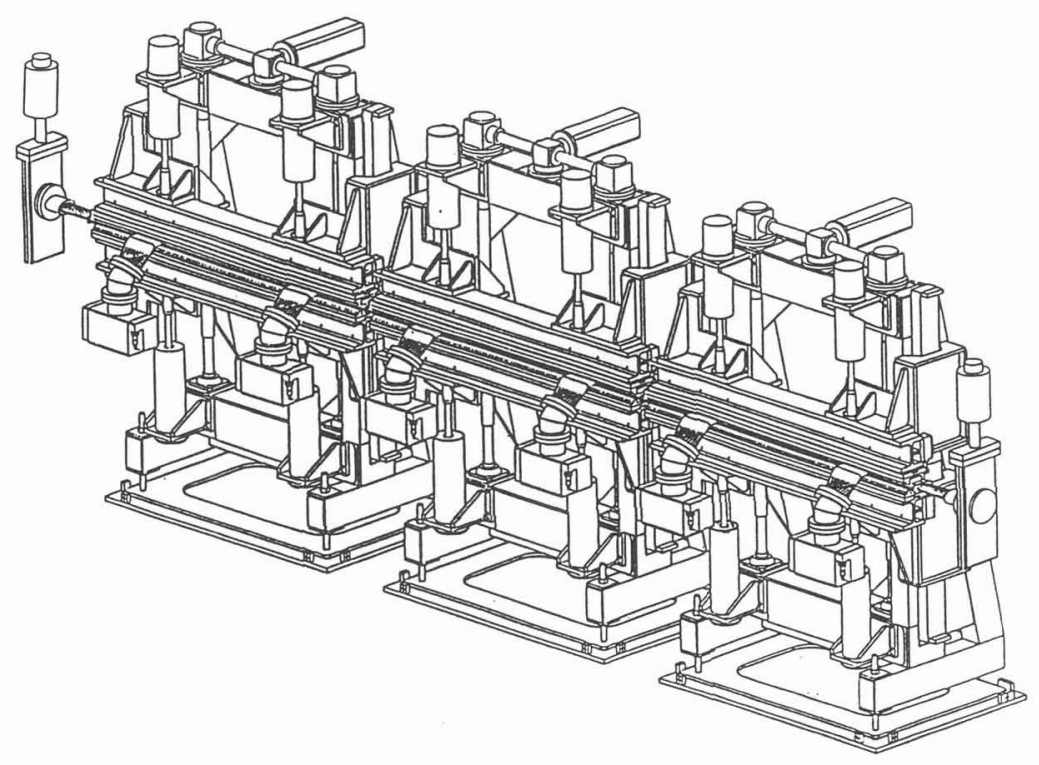

Figure 1: Straight section of the ESRF storage ring fully equipped with three segments of Insertion Devices.

Each segment is controlled independently from the other. The three segments can have identical or different magnetic fields according to the needs of the users of the beam line. If an identical magnetic field is required in all three segments, a proper phasing of each undulator segment is required, which can be a problem to the extent that some designers have preferred the use of a single long undulator carriage (ALS) and sacrificed the flexibility of the segmented approach.

The performance (Highest photon energy, tunability) of the undulators highly depends on the minimum achievable magnetic gap. Small magnetic gaps may limit the lifetime and the injection efficiency of the electron beam in the storage ring. A reasonable compromise must be made. In most existing projects, the total aperture left to the electron beam ranges from 10 to $20 \mathrm{~mm}$, to which one must add the thickness and tolerances of the vacuum chamber 4 to $5 \mathrm{~mm}$. As a result, a typical magnetic gap of 15 to $25 \mathrm{~mm}$ is usually achieved (20mm at ESRF). More ambitious and costly devices are envisaged with permanent magnet blocks placed in the vacuum in order to reduce the magnetic gap to the absolute minimum.

\section{RADIATION FROM INSERTION DEVICES}

\subsection{Undulators and Wigglers}

The spectral characteristics of ID strongly depend on the magnetic field. More precisely, one can define a so-called deflection parameter $\mathrm{K}$ :

$\mathrm{K}=0.0934 \operatorname{Per}[\mathrm{mm}] \mathrm{B}[\mathrm{T}]$ 
where Per is the spatial period of the magnetic field in $\mathrm{mm} . \mathrm{B}$ is the peak magnetic field in Tesla. $\mathrm{K}$ is dimensionless. For values of $\mathrm{K}$ smaller than 2 to 3 , the spectrum of the radiation presents a series of narrow peaks (see Figure 2).

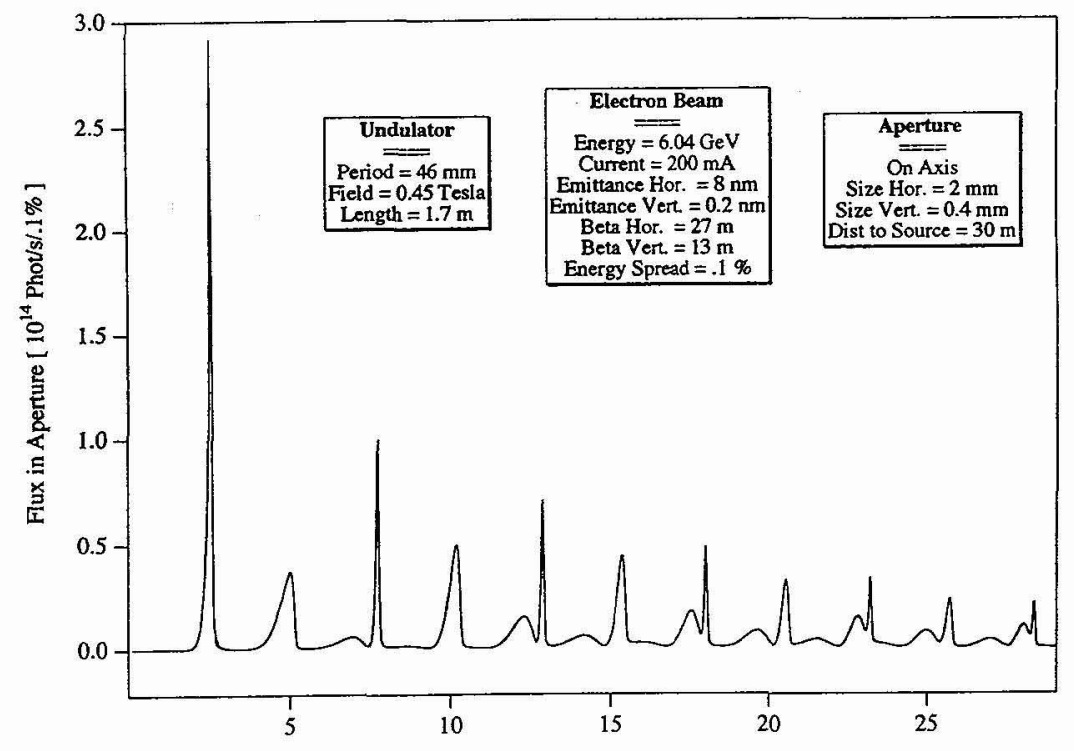

Figure 2: Computed undulator spectrum observed through a narrow aperture at ESRF.

Each peak originates from the constructive interference of the emission from each period. The radiation beam at the photon energy of these peaks is extremely brilliant. These devices are called "undulators" as opposed to "wigglers". The spectrum from a wiggler is just as smooth as the one from a bending magnet. High $\mathrm{K}$ values are obtained by combining large periods and/or high fields. Undulators generate extremely brilliant $\mathrm{x}$-ray beams at low and medium photon energies while wigglers extend the spectrum to much harder photon energies. The width of the peaks essentially depends on the emittance of the electron beam, beta function of the ring lattice and quality of the magnetic field.

The photon energy corresponding to the transition between the undulator and the wiggler domains scales like the square of the electron energy and depends on the minimum magnetic gap. At ESRF (6.04 GeV, $20 \mathrm{~mm}$ minimum gap) it reaches $30 \mathrm{keV}$ while a $1.5 \mathrm{GeV}$ machine would present the transition around 2 $\mathrm{keV}$. At ESRF nearly all undulators present a period smaller than $50 \mathrm{~mm}$ and a magnetic field smaller than $0.5 \mathrm{~T}$ while the wigglers present a field ranging from $0.7 \mathrm{~T}$ to $1.85 \mathrm{~T}$ with a period between 70 and $220 \mathrm{~mm}$

\subsection{Power}

The total power generated by an ID over the whole photon energy range and all directions of observation is : 
where $\mathrm{E}$ is the electron energy in $\mathrm{GeV}, \mathrm{L}$ is the length of the ID in meters and $\mathrm{I}$ is the ring current in amperes. A typical ESRF undulator presents a $0.5 \mathrm{~T}$ peak field, a length of $1.6 \mathrm{~m}$, it therefore produces 2 $\mathrm{kW}$ of radiation for a stored current of $200 \mathrm{~mA}$ and an electron energy of $6.04 \mathrm{GeV}$. Wigglers present a higher magnetic field and therefore produce higher power for the same length. A power as high as $15 \mathrm{~kW}$ is envisaged at ESRF.

\subsection{Power Density}

The power density generated on axis of an ID can be written in most cases of interest $(K>1)$ as :

$\mathrm{dP} / \mathrm{d} \Omega\left[\mathrm{kW} / \mathrm{mrad}^{2}\right]=10.8 \mathrm{~B}[\mathrm{~T}] \mathrm{E}^{4}[\mathrm{GeV}] \mathrm{I}[\mathrm{A}] \mathrm{N}$

where $\mathrm{N}$ is the number of periods of the ID (Length divided by the period). For a current of $200 \mathrm{~mA}$, a typical ESRF ID generates a power density of $53 \mathrm{~kW} / \mathrm{mrad}^{2}$ for a stored electron current of $200 \mathrm{~mA}$. Observed at normal incidence at a distance of $30 \mathrm{~m}$, it corresponds to a power density of nearly 60 $\mathrm{W} / \mathrm{mm}^{2}$.

\subsection{Brilliance}

An important figure of merit is the spectral brilliance namely the number of photons generated per second per $0.1 \%$ (of relative photon energy bandwidth) per $\mathrm{mm}^{2}$ of surface and per $\mathrm{mrad}^{2}$ of angular divergence. The next figure presents the brilliance of various representative ESRF undulators and wigglers.

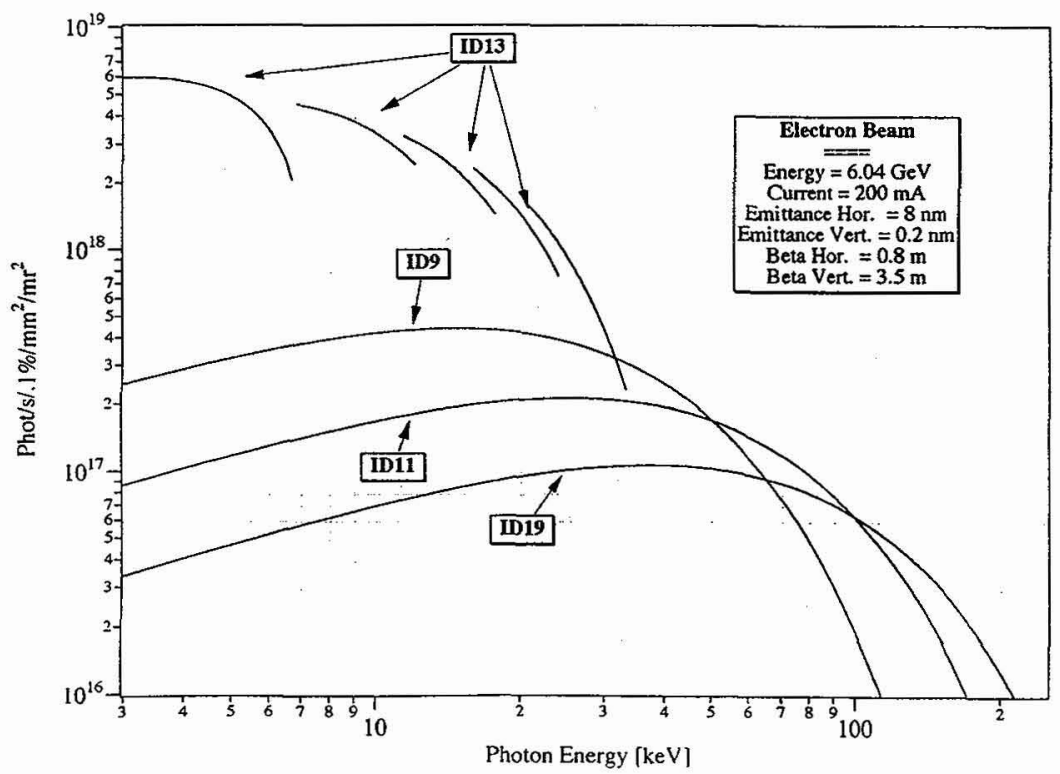


Figure 3: Spectral Brilliance of various representative Insertion Devices of the ESRF. ID13 (period = $46 \mathrm{~mm}$, Field $<0.48 \mathrm{~T}$ ) is a representative undulator similar to ID2-3-6-10. ID9, ID11 and ID19 are wigglers with peak field of $0.72 \mathrm{~T}, 1.25 \mathrm{~T}$ and $1.85 \mathrm{~T}$.

\subsection{Flux}

Some experiments integrate the radiation over a wide angle and/or surface. In that case a more relevant figure of merit is the spectral flux which simply represents the total number of photons generated by the ID within a small photon energy range. The next figure presents the spectral flux from various representative ESRF undulators and wigglers .

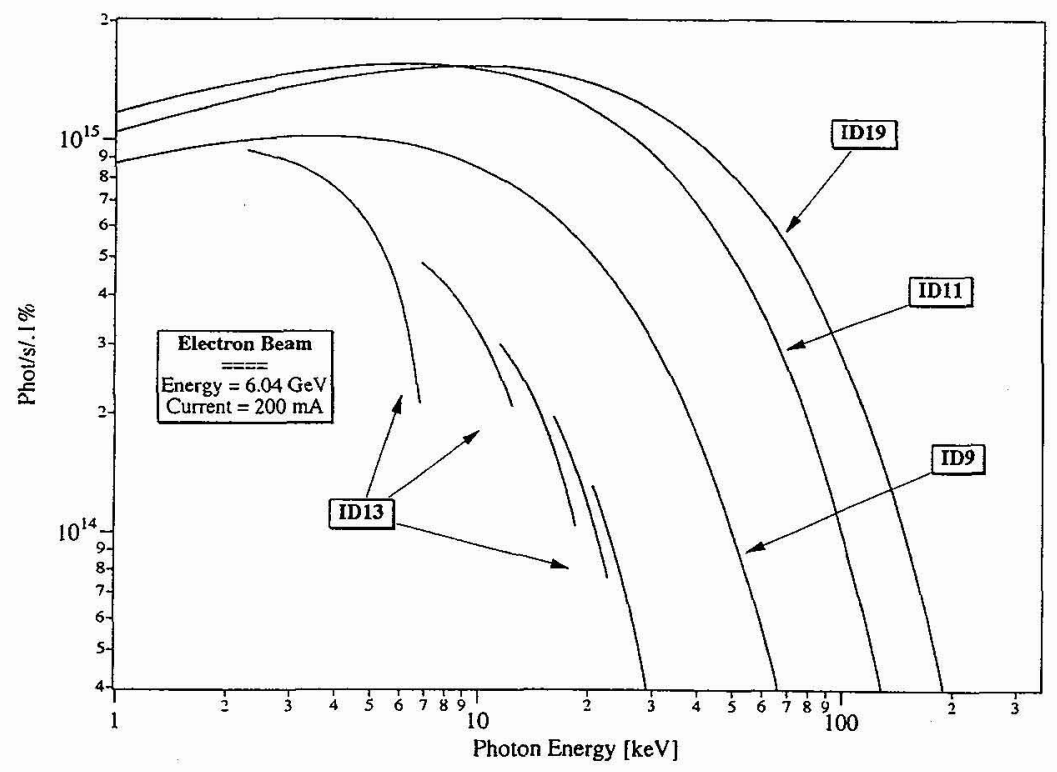

Figure 4: Spectral flux from various representative ESRF undulators and wigglers.

\subsection{Polarization}

The polarization of the radiation produced by the IDs depends on the direction of observation, the photon energy, the electron beam emittance and beta functions (ratio of the size over the divergence of the electron beam). Observed in the horizontal plane of the orbit, the radiation from wigglers is typically 90 $\%$ to $95 \%$ linearly polarized with electric field in the horizontal plane. Whichever direction of observation is selected, there is no net circular polarization (right and left handed components are generated equally). As one observes the radiation further away from the orbit plane, the radiation from a wiggler becomes more and more depolarized.

The radiation from undulators observed on axis of the electron at the photon energies of the peak of the harmonics is more than $99 \%$ linearly polarized in the horizontal plane. Away from the axis, the radiation is still mostly linearly polarized with a direction of the electric field depending on the direction of observation. Depolarization takes place essentially due to the non zero emittance. 


\section{CIRCULAR POLARIZATION}

A significant part of the user community is interested in circularly polarized x-ray radiation. Special IDs have been designed and built all over the world in order to satisfy this community.

\subsection{Undulators}

There are basically two kinds of undulator designs capable of producing brilliant circularly polarized radiation, namely the crossed undulator [2-3] and the helical undulator[4-6]. At high electron energy, the radiation from crossed undulators tends to be depolarized and helical undulators are the preferred source. A new kind of helical undulator[7] has been installed on the ID12 beam line of ESRF that is capable of producing Brilliant $95 \%$ circularly polarized radiation between 0.5 and $5 \mathrm{keV}$. Two other devices will be installed within a year that will cover the 2 to $10 \mathrm{keV}$ range.

\subsection{Wigglers}

If circularly polarized radiation is requested at photon energies not suitable for undulators $(>15 \mathrm{keV}$ for the ESRF), one must use the wiggler kind of radiation. There exists two modifications of the wiggler capable of producing circularly polarized radiation, namely the asymmetric wiggler [8] and the ellipsoidal wiggler [9]. Asymmetric wigglers are much simpler to design and build but generate a typical factor 2 smaller useful flux. At ESRF two asymmetric wigglers have been installed on ID15 and ID20 with a peak field of $1.85 \mathrm{~T}$ and $1.0 \mathrm{~T}$ capable of producing circularly polarized radiation in the 20 to 200 keV photon energy range.

\section{Conclusion}

The presentation given in this paper is largely inspired from the development made at ESRF. The Trieste ID group has been following very similar lines. The main options such as the segmentation and the shimming allow a series production of all kind of undulators and wigglers in a short time at a low price. Another approach has been followed at the ALS in Berkeley where hybrid technology has been used to build the U5 undulator. The coupling of 3 segmented hybrid undulators is a non trivial task if one wants to conserve good performances on high harmonic numbers. ALS therefore decided to build a single $5 \mathrm{~m}$ long support structure which is bulky and expensive. Such problems are absent in the pure permanent magnet assembly where phasing is a much easier task. One must also emphasize that, the lower the machine energy, the more important the phasing between the segments because of the less important role played by the emittance and the lower sensitivity to heatload.

\section{REFERENCES}

[1] J. Chavanne et al., Rev. Sci Instr. 63, 317 (1992)

[2] M. Moissev, M. Nikitin, N. Fedorov, Sov. Phys. J. 21, 332(1978)

[3] K.J. Kim, Nucl. Instr. and Methods A219, 425 (1986)

[4] B.M. Kincaid, J. Appl. Phys. 48, 2684 (1977).

[5] K. Halbach, Nucl. Instr. and Methods 169, 1 (1980)

[6] H. Onuki, Nucl. Instr. and Methods A246, 94 (1986) 
[7[ P. Elleaume, Nucl. Instr. and Methods A291, 371 (1990)

[8] J. Goulon, P. Elleaume, D. Raoux, Nucl. Instr. and Methods A254, 192 (1987)

[9] S. Yamamoto, H. Kawata, H. Kitamura, M. Ando, Phys. Rev. Lett. 62, 2672 (1989) 